\section{RB moonlights in mitosis}

Loss of the retinoblastoma (RB) tumour suppressor pathway commonly occurs in tumours and is associated with chromosome instability (CIN). The RB pathway is famous for its role in mediating G1/S cell cycle checkpoint control, but can this role alone account for its tumour suppressive function?

Evidence suggests that loss of the RB pathway affects mitosis, so Van Harn and colleagues arrested mouse embryonic fibroblasts in which the three $\mathrm{Rb}$ family members were ablated (TKO-MEFs) in G2 by serum starvation. This induced the cyclin-dependent kinase inhibitor p21, produced double-strand DNA breaks and activated the homologous recombination repair pathway. Inhibiting DNA damage response (DDR) kinases accelerated cell cycle re-entry and progression into mitosis after release from serum starvation and downregulated p21 expression. These data indicate that serumstarved RB pathway-deficient cells accumulate DNA damage during progression through the preceding $S$ phase and thereby activate DDR pathways. Moreover, metaphase spreads of TKO-MEFs showed defects in centromeric sister chromatid cohesion and more chromatid breaks than in controls, suggesting damage persists in mitosis. Consistent with this, the authors showed that serum-starved TKO-MEFs that re-entered the cell cycle after serum addition had increased copy number alterations. Sister chromatid cohesion is initiated during $\mathrm{S}$ phase by the cohesin complex, and although the authors did not find evidence of improper loading of the cohesin complex they suggested that this process might be defective when the $\mathrm{RB}$ pathway is inactivated.
Manning and colleagues used RNA interference to downregulate RB1 expression in TERTimmortalized RPE1 cells (which are diploid epithelial cells) and found that the loss of RB1 induces aneuploidy and chromosome missegregation. Knock down of RB1 did not affect proliferation but increased the mitotic index by twofold. In particular, the authors found a high proportion of cells in prometaphase, indicating that loss of RB1 delayed mitotic progression. On further analysis, the authors observed increased inter-centromeric distance between sister chromatids, defects in chromosome congression, sister kinetochore orientation and alignment on the metaphase plate. The authors proposed that these mitotic defects might originate from a defect in the centromere structure, which is regulated by the cohesin and condensin complexes. They found that chromatin association of RAD21 - a subunit of the cohesin complex - was reduced in mitotic cells lacking RB1, suggesting that its loss impairs the loading or maintenance of the cohesin complex at the centromere.

RBF1, the Drosophila melanogaster $\mathrm{RB}$ homologue, interacts with the condensin II subunit CAP-D3. Adding to these findings, Manning and colleagues showed that RBF1 also promotes cohesin complex loading onto DNA in D. melanogaster. In addition they found that CAP-D3 loading on chromatin was decreased in RB1deficient RPE1 cells and that knock down of CAP-D3 caused similar mitotic defects in RPE1 cells to those of RB1 knockdown. Interestingly, condensin II complexes are enriched at the centromeres of mitotic chromosomes, suggesting that RB1 loss causes defective centromeric condensation and cohesion, which leads to chromosome segregation errors and CIN.

So, is this role of RB1 important for its function as a tumour suppressor? Coschi et al. used $R b 1^{\Delta \mathrm{L} / \Delta \mathrm{L}}$ mice that express a mutant RB1 that cannot bind condensin II complexes to test this. The authors showed that the increased lagging chromosomes and centromeric fusions observed in MEFs from these mice were not the result of loss of G1/S cell cycle checkpoint control. Live imaging of these MEFs revealed the same mitotic defects, which they showed caused aneuploidy from either an abrupt termination of the delay at metaphase (probably caused by chromosome breakage) or a failure to complete mitosis (which generated binucleated cells). Condensin II subunits were also improperly loaded onto chromatin. The expression of the RB1 mutant reduced the latency of tumour initiation in $R b 1^{\Delta \mathrm{L} / \Delta \mathrm{L}} ; \operatorname{Tr} p 53^{-/-}$ and $R b 1^{\Delta \mathrm{L} / \Delta \mathrm{L}} ; \operatorname{Tr} p 53^{+/-}$mice. Moreover, the tumours were more aggressive, there was a higher incidence of metastases and a higher proportion of mice with multiple tumours. By comparing thymic lymphoma cells from $R b 1^{\Delta \mathrm{L} / \Delta \mathrm{L}}$; $\operatorname{Tr} p 53^{-/-}$and $\operatorname{Tr} p 53^{-/-}$mice the authors found that whole and segmental chromosome gains and losses were increased in the lymphoma cells that expressed mutant RB1, suggesting that the accompanying CIN could account for the reduced tumour latency.

Together, these data suggest that the CIN resulting from the inability of RB1 to maintain sister chromatid cohesion promotes tumorigenesis and that this is an important function of RB1 as a tumour suppressor.

\section{Gemma K. Alderton}

ORIGINAL RESEARCH PAPERS van Harn, T. et al. Loss of RB proteins causes genomic instability in the absence of mitogenic signalling. Genes Dev. 24, 1377-1388 (2010) | Manning, A. L. et al. Loss of pRB causes centromere dysfunction and chromsome instability. Genes Dev. 24, 1364-1376 (2010)| Coschi, C. H. et al. Mitotic chromosome condensation mediated by the retinoblastoma protein is tumor suppressive. Genes Dev. 24, 1351-1363 (2010) 